\title{
Optical excitations and thermoelectric properties of two-dimensional holey graphene
}

\author{
Deobrat Singh $\odot,{ }^{1, *}$ Vivekanand Shukla $\odot,{ }^{1, \dagger}$ and Rajeev Ahuja $\odot^{1,2}$ \\ ${ }^{1}$ Condensed Matter Theory Group, Materials Theory Division, Department of Physics and Astronomy, \\ Uppsala University, Box 516, 75120 Uppsala, Sweden \\ ${ }^{2}$ Applied Materials Physics, Department of Materials and Engineering, Royal Institute of Technology (KTH), S-100 44 Stockholm, Sweden
}

(Received 15 May 2020; revised 15 July 2020; accepted 3 August 2020; published 26 August 2020)

\begin{abstract}
Recently, holey graphene (HG) was synthesized successfully at atomic precision with regard to hole size and shape, which indicates that HG has interesting physical and chemical properties for energy and environmental applications. The shaping of the pores also transforms semimetallic graphene into semiconductor HG, which opens new doors for its use in electronic applications. We investigated systematically the structural, electronic, optical, and thermoelectric properties of HG structure using first-principles calculations. HG was found to have a direct band gap of $0.65 \mathrm{eV}$ (PBE functional) and $0.95 \mathrm{eV}$ (HSE06 functional); the HSE06 functional is in good agreement with experimental results. For the optical properties, we used single-shot $G_{0} W_{0}$ calculations by solving the Bethe-Salpeter equation to determine the intralayer excitonic effects. From the absorption spectrum, we obtained an optical gap of $1.28 \mathrm{eV}$ and a weak excitonic binding energy of $80 \mathrm{meV}$. We found large values of thermopower of $1662.59 \mu \mathrm{V} / \mathrm{K}$ and a better electronic figure of merit, $Z T_{e}$, of 1.13 from the investigated thermoelectric properties. Our investigations exhibit strong and broad optical absorption in the visible light region, which makes monolayer $\mathrm{HG}$ a promising candidate for optoelectronic and thermoelectric applications.
\end{abstract}

DOI: 10.1103/PhysRevB.102.075444

\section{INTRODUCTION}

The first acknowledged isolation and discovery of singlelayer graphene by backward exfoliation of graphite was accomplished in 2004 by Geim and co-workers [1,2]. Since then, significant efforts to study the captivating attributes, applications, and synthesis methods of graphene have led to new research directions [3,4]. Graphene consists of a monolayer of carbon atoms in a honeycomb arrangement with $s p^{2}$-hybridized carbon atoms that exhibit unusual and interesting electrical, optical, thermal, and mechanical characteristics. Along with other properties, graphene has resulted in increased research attention in order to determine its applicability on a realistic scale [5-7]. It is well understood that graphene manifests high electrical conductivity [8], a frictionless surface [9], a high ambipolar electric field [10], and quantum Hall effects at room temperature [11]. In addition, graphene is believed to be the strongest material ever known to humankind, and it exhibits high carrier mobility [12-14]. Graphene is an excellent candidate for transparent and conductive composites, electrodes, and photonics

\footnotetext{
*deobrat.singh@physics.uu.se

†Present address: Department of Microtechnology and Nanoscience (MC2), Chalmers University of Technology, SE-41296 Gothenburg, Sweden; vns391@gmail.com
}

Published by the American Physical Society under the terms of the Creative Commons Attribution 4.0 International license. Further distribution of this work must maintain attribution to the author(s) and the published article's title, journal citation, and DOI. Funded by Bibsam. applications [12]. However, despite having all these unique properties, graphene possesses a zero-gap electronic structure. As a result, there are limitations to its use in some electronic applications, such as transistors, for which it exhibits extremely high off-state current and a very low on/off ratio $[12,15]$. The positive effect of this particular limitation is that it served as motivation for scientific endeavors during the past decade to synthesize, design, and fabricate novel twodimensional (2D) materials [16-18]. As a result, we now have several 2D materials with a variety of different properties and applications. Apart from this, several physical and chemical strategies, such as oxidation, hydrogenation, and fluorination, have been attempted in order to achieve an electronic gap in graphene pertaining to its high mobility [19-22].

In recent years, the perforation of $2 \mathrm{D}$ materials has emerged as an effective way to enhance their electronic, mechanical, and optical properties, and to broaden their applicability in ways that go beyond their pristine structure [23-29]. Perforated graphene, which is also known as holey graphene (HG), turns out to be permeable due to its small holes. This permeability, combined with its intrinsic strength and layered thickness, suggests that graphene may have future applications as the most flexible and selective filter for tiny substances, including biomolecules, greenhouse gases, and salts [30-32]. When the spacing between holes is repeatedly reduced to a few atoms, the electronic structure of graphene is transformed from a semimetal to a semiconductor [33]. There have been several theoretical reports on various HGs with different pore sizes [34-37]. HG can have astonishing properties due to the quantum confinement effect, although the controlled experimental realization of $\mathrm{HG}$ with finite perforation remains a challenging task. Recently, Moreno and 
co-workers devised a way to synthesize holey graphene with outstanding precision using bottom-up method [38]. Apart from this, they were also successful at transferring it to a dielectric substrate, which enhances the possibility of using HG for possible device applications, where it can replace the bulkier, more rigid silicon components used today.

Taking this as motivation, in the present work we have studied the structural, electronic, optical, and thermoelectric properties of a HG monolayer with hybrid functional HSE06. We also investigated the $G_{0} W_{0}+\mathrm{BSE}$ method for excitonic optical properties, since to our knowledge there is no work that has been done on this $\mathrm{HG}$ with $G_{0} W_{0}+\mathrm{BSE}$, hence our work is timely. The $G W+\mathrm{BSE}$ approach is much more accurate for the optical properties of the excitonic effect due to its quasiparticle (QP) treatment. Previous investigations [38] suggest that the QP band gap of a HG monolayer is overestimated as compared to the experimental band gap. In this study, the HSE06 functional gives a more accurate band gap and it is consistent with experimental work, and band dispersion suggests that HG has anisotropic electronic and transport properties. We found that there are strong optical absorptions in the visible light region for a $\mathrm{HG}$ monolayer and weak excitonic binding energies. Also, for the thermoelectric properties, we have calculated the Seebeck coefficient (thermopower), the electrical conductivity, the electronic thermal conductivity, and the electronic figure of merit. The electronic figure of merit $Z T_{e}$ reaches up to 1.13. The Seebeck coefficient and $Z T_{e}$ are significantly higher than the pristine graphene monolayer and most of the $2 \mathrm{D}$ materials. These features are promising due to the sharp density of states (DOS) around the Fermi level, which occurs due to the quantum confinement effects of nanopores. Our results suggest that novel 2D planar HG has better visible light absorption, which makes $2 \mathrm{D} \mathrm{HG}$ a promising candidate for potential applications in the field of optoelectronic and thermoelectric devices.

This paper is organized as follows. In Sec. II, we describe the computational methods employed in the present work. In Sec. III A, we study the structural and electronic properties of a HG monolayer. Section III B shows the excitonic effect, and it contains an investigation of the optical proprieties of a $\mathrm{HG}$ monolayer. Finally, based on electronic transport, Sec. III C explores the thermoelectric properties of a $\mathrm{HG}$ monolayer.

\section{COMPUTATIONAL METHOD}

Electronic structure is based on first-principles calculations, and we employed the plane-wave basis projector augmented wave (PAW) method in the framework of densityfunctional theory (DFT) [39]. For the exchange-correlation potential, the generalized gradient approximation (GGA) in the form of Perdew-Burke-Ernzerhof (PBE) was employed as implemented in the Vienna Ab Initio Simulation Package (VASP) software [40]. The inherent underestimation of the band gap given by DFT within the inclusion of a hybrid functional is corrected by using the Heyd-Scuseria-Ernzerhof (HSE06) [41] screened-nonlocal-exchange functional of the generalized Kohn-Sham scheme. The charge transfer in the structures was determined by the Bader analysis [42]. The energy cutoff value for the plane-wave basis set was taken to be $500 \mathrm{eV}$. The total energy was minimized until

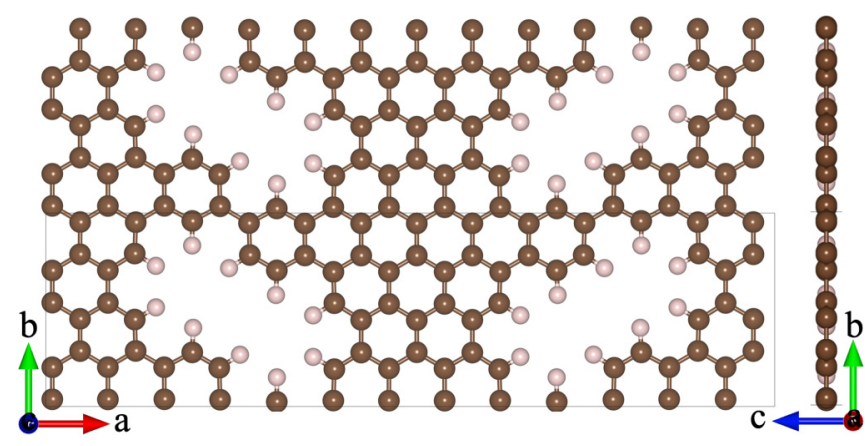

FIG. 1. Schematic representation of the optimized holey graphene with top and side views.

the energy variation in successive steps became less than $10^{-6} \mathrm{eV}$ in the structural relaxation, and the convergence criterion for the Hellmann-Feynman forces was taken to be $10^{-3} \mathrm{eV} / \AA . \AA .2 \times 4 \times 1 \Gamma$-centered $k$-point sampling is used for the primitive unit cell. The Gaussian broadening for the density-of-states calculation was taken to be 0.10 .

The dielectric function and the optical oscillator strength of the HG monolayer were calculated by solving the BetheSalpeter equation (BSE) on top of the single-shot $G_{0} W_{0}$ calculation, which was performed instead of standard DFT calculations $[43,44]$. The $G_{0} W_{0}+\mathrm{BSE}$ approach accounted for both $e-e$ and $e-h$ effects. Here, $e-e$ and $e-h$ represent the electron-electron and electron-hole correlation effects, respectively. During this process, we used $2 \times 4 \times 1 \Gamma$-centered $k$-point sampling. The cutoff for the response function was set to $250 \mathrm{eV}$. The number of bands used in our calculations is 340. The cutoff energy for the plane waves was chosen to be $400 \mathrm{eV}$. We included 36 valence (occupied orbitals) and 72 conduction (unoccupied orbitals) bands in the calculations for the dielectric function of HG monolayers in the BSE calculations. In previous investigations, 96 empty states converged for a MoS2 monolayer [45], and 150 empty states were taken for $G W$ calculations for a bulk system with semiconductors and insulators by Shishkin and co-workers [46].

Further, the electron transport properties are computed by using semiclassical Boltzmann transport theory with the relaxation time approximation and the rigid band approximation as implemented in the BoLTzTrAP code [47]. From the thermoelectric properties, we obtain the Seebeck coefficient (thermopower) $\alpha$, which is independent of the relaxation time $\tau$, electrical conductivity $\sigma$, and electronic thermal conductivity $\kappa_{e}$, which depends on the relaxation time $\tau$. To evaluate the thermoelectric properties, we use the electronic figure of merit, $Z T_{e}=S^{2} \sigma T / \kappa_{e}$, which shows the characteristics of electron transport and gives the upper limit of the total $Z T$.

\section{RESULTS AND DISCUSSION}

\section{A. Structural and electronic properties}

The optimized structure of a HG monolayer is depicted in Fig. 1, which shows that $\mathrm{HG}$ has a planar structure (top and side views). As can be seen in Fig. 1, the catafused benzene rings result in a periodic modulation of the width, and consecutive pairs of 7 and 13 carbon atoms, which is the precise edge structure of the nanoribbons, referred to as 


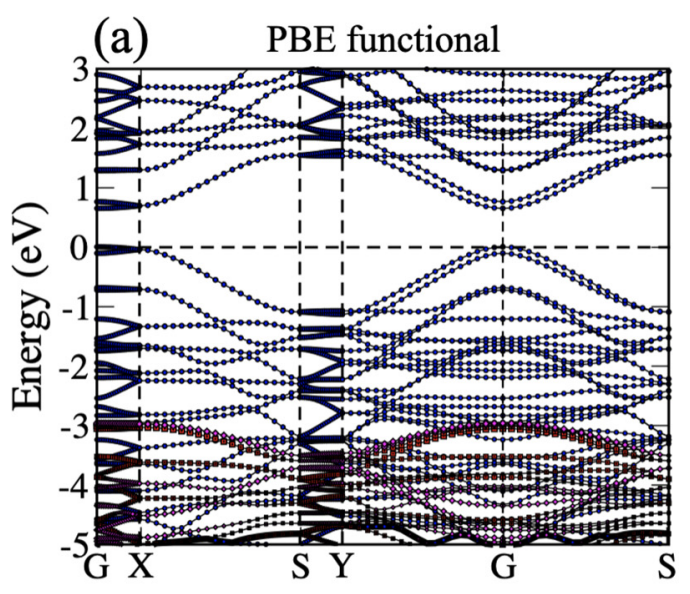

(b) HSE06 functional

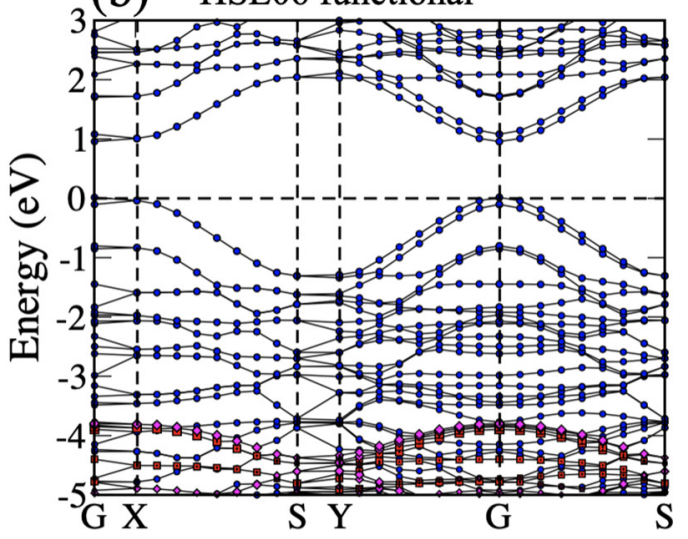

FIG. 2. Electronic band structures with orbital projection: (a) PBE functional and (b) HSE06 functional. Blue, red, magenta, and green represent the $p_{z}, p_{y}$, and $p_{x}$ states from the $\mathrm{C}$ atom and the $s$ state from the $\mathrm{H}$ atom, respectively.

7-13 AGNR. This further defines the size of the corresponding pores in the HG. Due to the presence of two asymmetric pores with different orientations, $\mathrm{HG}$ has a large unit cell with lattice parameters of $a=32.38 \AA$ and $b=8.58 \AA$. The pores are made of two benzene rings and they became terminated with hydrogen atoms. Also, the bond lengths between C-C and C-H are 1.38-1.43 and $1.09 \AA$, respectively, and they are consistent with previous works $[48,49]$.

The electronic band structure is presented in Fig. 2(a) using the PBE functional. It is evident that it has a direct band gap of $0.65 \mathrm{eV}$ at the $\Gamma$ point. The computed band gap is in good agreement with previously investigated works [38,48-50]. This band of the HG structure depends on the thickness of the interconnects, which is clearly governed by the nanoribbon width. The band gap will increase with a decrease of the nanoribbon width and vice versa. One can see that the $p_{z}$-orbital of the $\mathrm{C}$ atom mainly contributes near the Fermi level, $E_{F}$, in the valence-band maximum (VBM) and also in the conduction-band minimum (CBM) (see Fig. 2). States belonging to the $\sigma$ bond, $p_{x}$ and $p_{y}$, are deep down in the Fermi level, and they can be seen around $-3 \mathrm{eV}$. No contribution from the hydrogen $s$-orbitals appears near the VBM and CBM region. It is evident that HG has a directional-dependent electronic structure. $G-X$ band lines

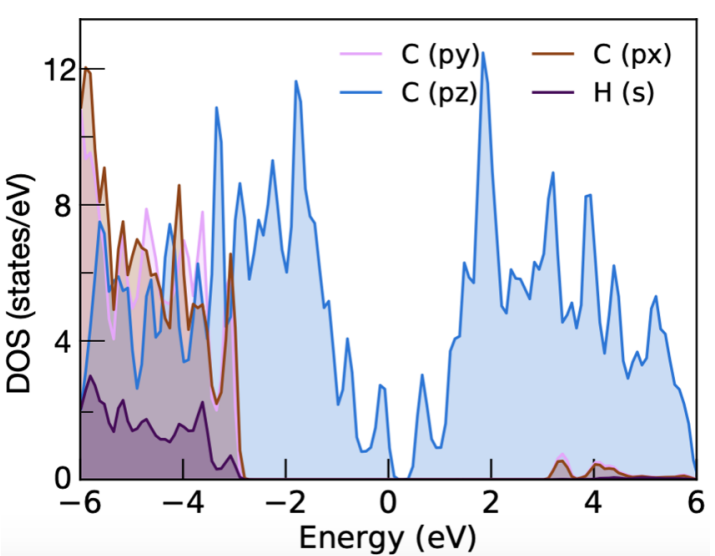

FIG. 3. Decomposed orbital density of states of a HG monolayer with a PBE functional.

have a nearly flat dispersion in comparison to $G-Y$ band lines both in conduction and valence bands. PBE generally underestimates the band gap of materials because it is singleparticle, hence to find a more accurate electronic band structure we use a hybrid functional HSE06, which involves 25\% of Fock exchange. The HSE06 functional yields a band gap of $0.95 \mathrm{eV}$ as shown in Fig. 2(b), which is well matched with the experimentally reported band gap of this perforated monolayer. However, the anisotropic nature of the electronic structure persists, and the dispersion around the VBM and CBM remains the same.

The decomposed orbital density of states for a HG monolayer is displayed in Fig. 3 showing three subbands: (a) the lower valence bands $(<-3 \mathrm{eV})$ mainly dominated by C- $p_{x}$, $p_{y}$, and $\mathrm{H}-s$ states; (b) the subbands dominated by C- $p z$ states near (valence band) and above (conduction band) the Fermi level; and (c) the strongly hybridized C- $p_{x}, p_{y}$, with $\mathrm{H}-s$ subbands $(>3 \mathrm{eV})$. This information is crucial and will help during the interband transitions from the VBM to the CBM. The possible interband transition originates mainly from (i) $\pi \rightarrow \pi^{*}$ with an energy range of -3 to $+3 \mathrm{eV}$, and (ii) $\pi \rightarrow$ $\sigma^{*}, \sigma \rightarrow \pi^{*}$, and $\sigma \rightarrow \sigma^{*}$ for other energy ranges. In the following subsection (Sec. III B), we will discuss the optical properties. Before moving to the possible optical transition, we have also calculated the effective mass and carrier mobility of the electron and hole in a HG monolayer. The simulated effective masses of the electrons $\left(m_{e}^{*} / m_{0}\right)$ and holes $\left(m_{h}^{*} / m_{0}\right)$ for the CBM and VBM of the HG monolayer are defined as

$$
m^{*}=\hbar^{2}\left[\frac{\partial^{2} E(k)}{\partial k^{2}}\right]^{-1}
$$

where $\hbar, k$, and $E(k)$ represent the reduced Planck constant, the wave vector, and the respective energy dispersion in the CBM and the VBM. Using the above equation, the effective masses are estimated as $0.40 m_{0}$ and $0.09 m_{0}$ for electrons and $0.40 m_{0}$ and $0.08 m_{0}$ for holes in the $a$ - and $b$-directions, respectively. In the literature, the effective mass of an electron in a graphene monolayer was found to be $0.012 m_{0}$ [51]. Moreover, the theoretical carrier mobility for a better understanding of the electronic conductance of a HG monolayer is calculated by using deformation potential (DP) theory devised by Bardeen 
TABLE I. Theoretically calculated electron and hole carrier mobility $\mu\left(\mathrm{cm}^{2} \mathrm{~V}^{-1} \mathrm{~s}^{-1}\right)$ using deformation potential (DP) theory at room temperature.

\begin{tabular}{lrcc}
\hline \hline System & \multicolumn{1}{c}{$\mu_{e}$} & $\mu_{h}$ & Ref. \\
\hline Graphene & $33.89 \times 10^{4}$ & $32.17 \times 10^{4}$ & {$[54]$} \\
$\alpha$-CP & $38.70 \times 10^{2}$ & $11.51 \times 10^{4}$ & {$[55]$} \\
$\beta$-CP & $12.910 \times 10^{3}$ & $15.52 \times 10^{3}$ & {$[55]$} \\
BP & $22.0 \times 10^{2}$ & $10.0 \times 10^{3}$ & {$[56,57]$} \\
Graphyne & $42.90 \times 10^{4}$ & $54.10 \times 10^{4}$ & {$[58]$} \\
HG $(a$-dir $)$ & $23.77 \times 10^{1}$ & $12.58 \times 10^{2}$ & This work \\
HG $(b$-dir $)$ & $13.56 \times 10^{2}$ & $14.18 \times 10^{3}$ & This work \\
\hline \hline
\end{tabular}

and Shockley [52]. The carrier mobility can be calculated by the following relation [53]:

$$
\mu=\frac{2 e \hbar^{3} C}{3 k_{B} T\left|m_{*}\right|^{2} E_{1}^{2}},
$$

where $C$ is the elastic modulus, which is calculated by a quadratic fitting of the energy-strain data; $k_{B}$ and $T$ are the Boltzmann constant and temperature; and $m^{*}$ is the effective mass calculated by using the previous equation. $E_{1}$ is the deformation potential $\left[E_{1}=\Delta E(\Delta a / a)\right]$, in which $\Delta E$ is the energy shift in the valence-/conduction-band edge with respect to the lattice variation $(\Delta a / a)$. The calculated electron and hole carrier mobilities are reported in Table I for both the $a$ - and $b$-directions, and we see the anisotropy in mobilities of order 1 . The carrier mobility of HG is found to be lower than that of the graphene, carbon phosphide, graphyne and black phosphorus (see Table I).

The carrier mobility of HG is lower than that of the pristine graphene monolayer, which is $(3-4) \times 10^{5} \mathrm{~cm}^{2} \mathrm{~V}^{-1} \mathrm{~s}^{-1}$ for the electron and hole [54,58,59]. This is good for nanoelectronic device application because graphene shows a poor on/off ratio due to its extremely high mobility and semimetallic nature.

\section{B. Optical properties}

In this subsection, applying the $G W+$ BSE method, we discuss the optical properties of a HG monolayer, such as the real $[\operatorname{Re}(\epsilon)]$ and imaginary $[\operatorname{Im}(\epsilon)]$ parts of the dielectric function, the anisotropic nature of $\operatorname{Im}(\epsilon)$, the complex refractive index, absorption spectra, optical conductivity, the electron energy-loss spectrum (EELS), and reflectivity. The $e-e$ correlation is considered to be the imaginary part $\operatorname{Im}(\epsilon)$ of the dielectric function for HG by using $G_{0} W_{0}$ plus BSE functions. $G_{0} W_{0}$ plus BSE establish a higher-order interaction illustration, i.e., $e-e$ and $e-h$ effects are considered to show an accurate electronic description systematically on top of $G_{0} W_{0}$. Additionally, the $e$ - $h$ interaction produces mainly a renormalization of the intensity of the optical peaks computed using the $G_{0} W_{0}$ plus BSE method. The imaginary part of the dielectric functions acquired at the $G_{0} W_{0}$ plus BSE levels for HG is presented in Fig. 4, which shows that the inclusion of both $e-e$ and $e-h$ interactions yields a noteworthy redshift, which is consistent with the previous results [60]. Another appealing and peculiar result is that the first BSE optical peak is in much better agreement with the electronic gap obtained

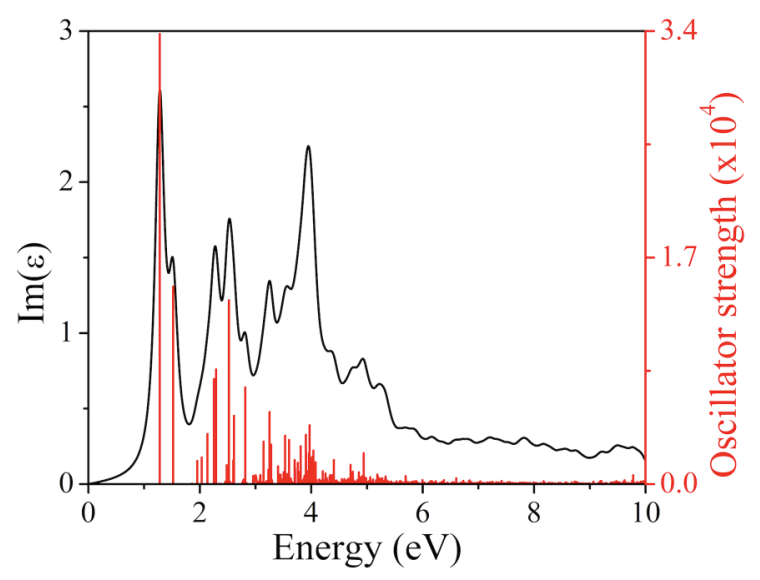

FIG. 4. Imaginary part of the dielectric function (black line) and the oscillator strength of the optical transitions (red bars) of holey graphene.

by the $G W$ method, implying the weakly bound excitonic/free carrier nature of the optical excitation. Consequently, the physical effect of the $e-e$ and $e-h$ interactions, reproduced by the $G_{0} W_{0}$ plus BSE method, provides a more accurate result.

To analyze the optical properties of the HG monolayers, the imaginary part of the dielectric function and the corresponding oscillator strength of the optical transitions of the HG monolayer are depicted in Fig. 4. In this case, we have two excitonic states with a very strong oscillator strength. The starting two peaks appear at 1.28 , and $1.52 \mathrm{eV}$ in the optical spectrum of the HG monolayer (see Fig. 4), originating primarily from the optical transitions at the high symmetry $\Gamma$ point in the BZ. The second peak split with $0.24 \mathrm{eV}$ of optical transitions also originates at the same high symmetry $\Gamma$ point. It was reported that the band gap is $1.36 \mathrm{eV}$ using self-energy corrections within the $G W$ method by Moreno and co-workers [38]. According to those authors, the excitonic binding energy (EBE) of a HG monolayer is found to be $80 \mathrm{meV}$, which is calculated by $\mathrm{EBE}=E_{g}^{G W}-E_{g}^{\text {optical }}$. The first absorption peak is correlated with the bound excitonic state, which is due to the intraband transition between the valence and the conduction band at high symmetry $G$. The important binding energy of an exciton in the case of a HG monolayer can efficiently open both electrons and holes, and thus suppress the rapid recombination of holes and photogenerated electrons, indicating that the HG monolayer has the possibility of being used in optoelectronic device applications. The calculated exciton binding energy for a HG monolayer is almost the same as bilayer graphene $(\approx 80 \mathrm{meV})$ [61], while a single layer of graphene $(270 \mathrm{meV})$ [61] is greater than a HG monolayer. Moreover, with regard to graphene derivatives, graphyne showed an exciton binding energy of $\approx 400 \mathrm{meV}$ [62] and other 2D materials such as $0.96 \mathrm{eV}$ in $\mathrm{MoS}_{2}$ [45] and $780 \mathrm{meV}$ in black phosphorus (BP) [63] and thus less Coulomb screening in a HG monolayer.

It is clear that the optical anisotropy among $E\|X, E\|$ $y$, and $E \| z$ largely increases due to the inclusion of local field effects, as shown in Fig. 5. The anisotropic results shown along $E \| x$ and $E \| y$ mean "in-plane"; the allowed optical transitions are observed at $1.28 \mathrm{eV}(\alpha), 1.52 \mathrm{eV}(\beta)$, 


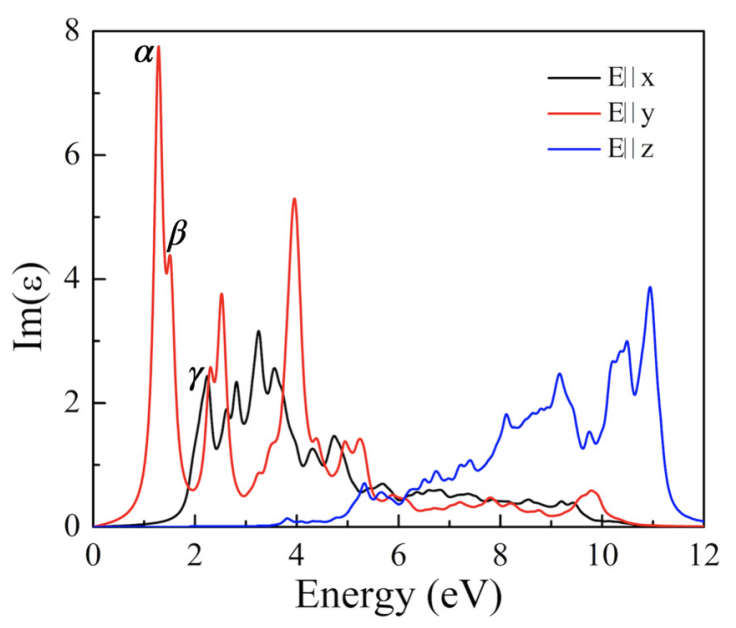

FIG. 5. The imaginary part of the dielectric function $E\|X, E\|$ $y$, and $E \| z$ corresponding to the holey graphene monolayer along the $x, y$, and $z$ directions, respectively. The black, red, and blue lines represent the imaginary components along the $x, y$, and $z$ directions, respectively. The $z$ component is the multiple of 100 on the $y$-axis data. and $2.24 \mathrm{eV}(\gamma)$. According to optical selection rules, only $\pi \rightarrow \pi^{*}$ and $\sigma \rightarrow \sigma^{*}$ transitions are allowed if the light is polarized parallel to the planar directions. This means that the mentioned peaks occur from the $\pi \rightarrow \pi^{*}$ transition. Moreover, along the $z$ direction, i.e., $E \| z$, out-of-plane, the allowed optical transitions appear at 3.8 and $5.32 \mathrm{eV}$. These transition peaks originate mainly from $\pi \rightarrow \sigma^{*}$ or $\sigma \rightarrow \pi^{*}$ transitions, which is allowed for the perpendicular polarization direction, i.e., $E \| z$, out-of-plane. Such types of spectra are useful as they provide valuable information on the optical transition probability corresponding to a certain light wavelength. It was also seen that the electronic band structure exhibits anisotropy behavior because the band dispersion profile is different along the high-symmetry point $G-X$ and $G-Y$. This means that the possibility of the creation of electrons and holes depends on the band dispersion profile with the incident of electromagnetic radiation on a HG monolayer [64]. According to that, the excitation will appear at a different photon energy along the $x$ and $y$ directions (see Fig. 5) due to their anisotropic dispersion of band structure as presented in Fig. 2. Consequently, optical absorption shows highly anisotropic behavior for optical excitations [65].

Furthermore, we compared the computed optical properties (i) without taking into account the $e-e$ and $e-h$ correlation, $\mathrm{PBE}+\mathrm{RPA}[66]$, (ii) with the incorporated $e-e$ inter-
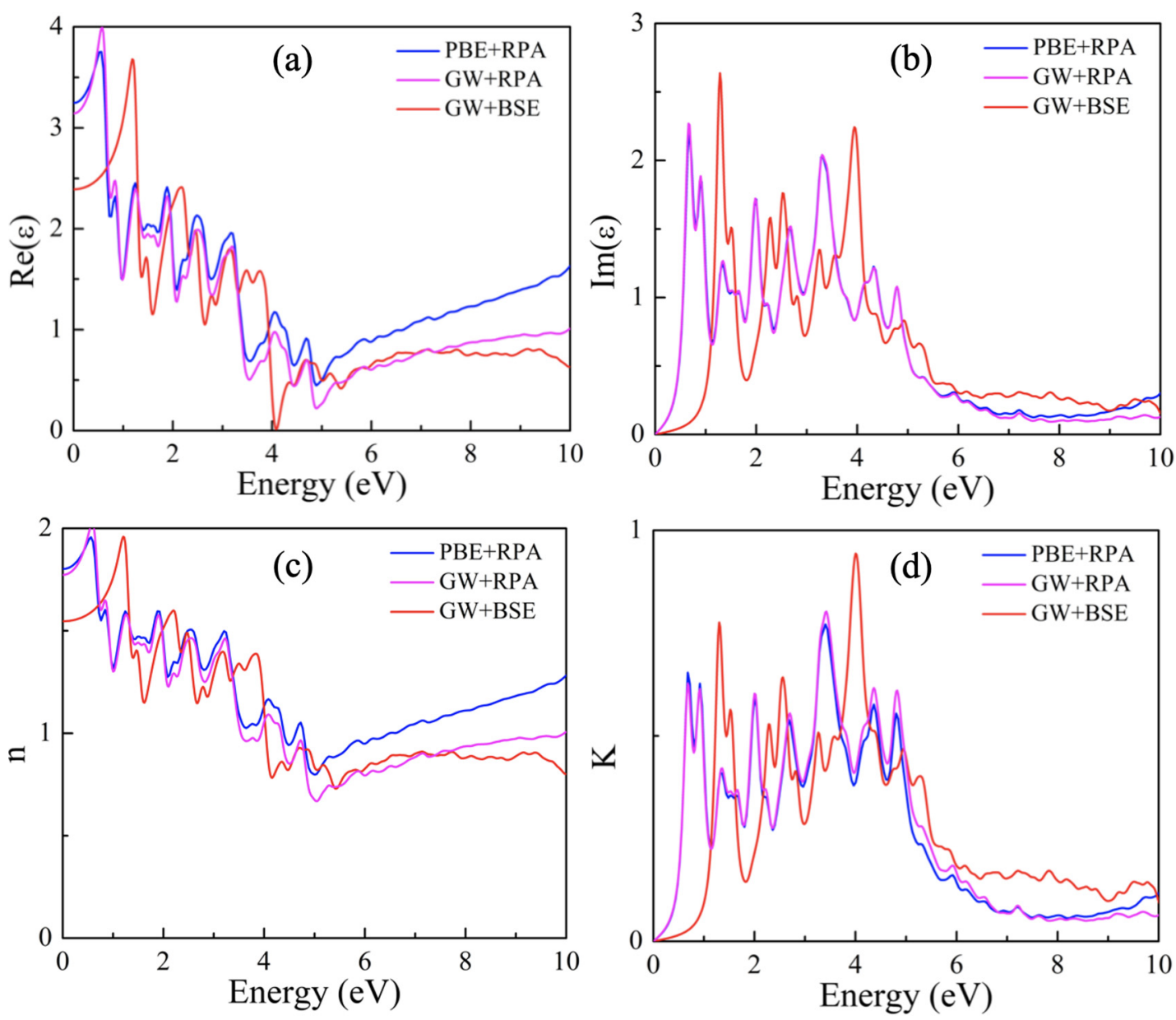

FIG. 6. Optical properties of holey graphene: (a) real part of the dielectric function, (b) imaginary part of the dielectric function, (c) refractive index, and (d) extinction coefficient with a comparison of the PBE functional and the $G W+\mathrm{BSE}$ method. 

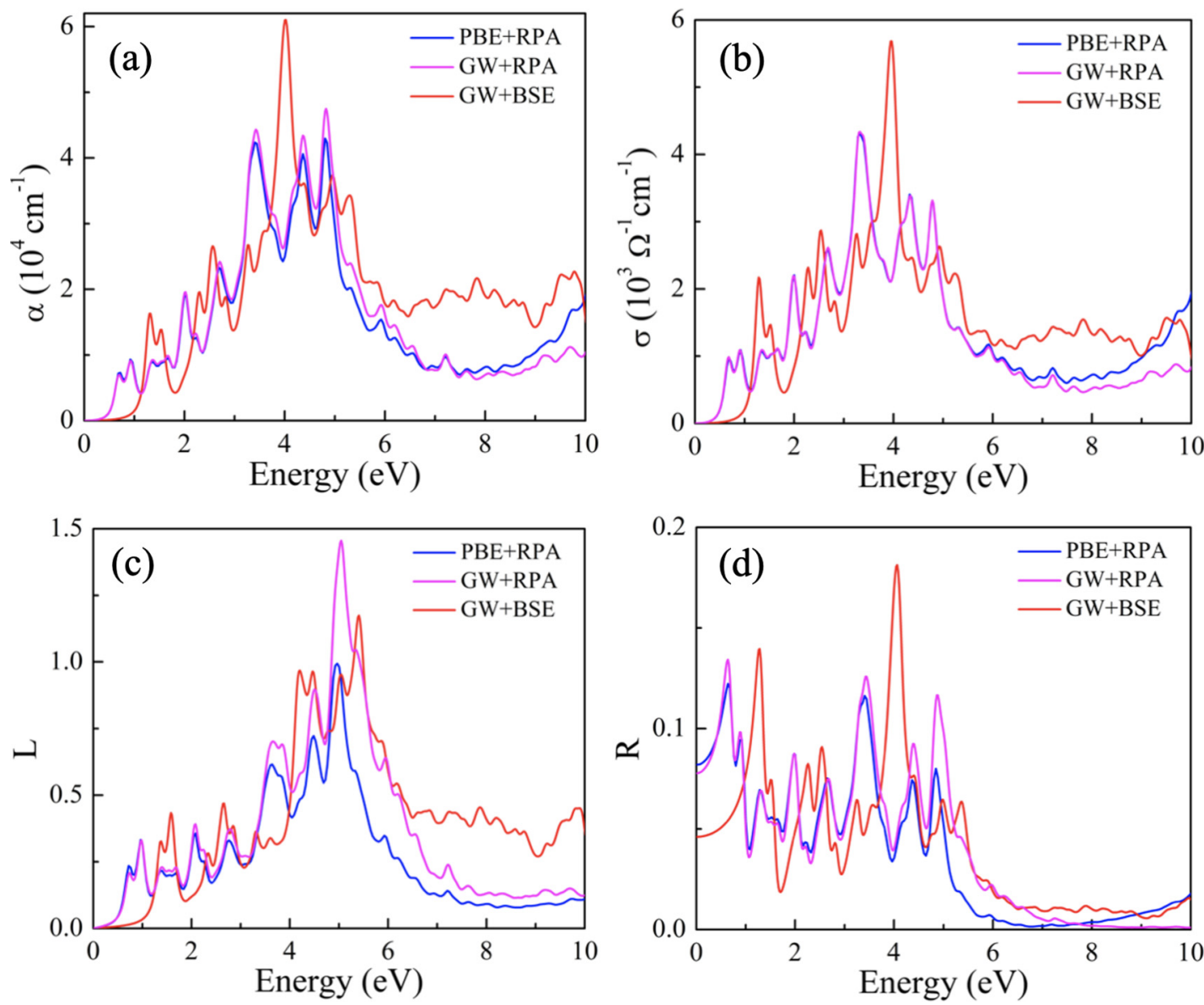

FIG. 7. Optical properties of holey graphene: (a) absorption spectra, (b) optical conductivity, (c) electron energy loss function, and (d) reflectively with a comparison of the PBE functional and the $G W+\mathrm{BSE}$ method.

action and neglected $e-h$ interactions, $G W+$ RPA [43], and eventually (iii) the $e-e$ and $e-h$ interactions, relevant in the photoexcitation processes, are associated with solving the Bethe-Salpeter equation (BSE) [67]. The real and imaginary parts of the dielectric function, refractive index, extinction coefficient, optical absorption, optical conductivity, EELS, and reflectivity of the HG monolayer obtained using PBE+RPA, $G_{0} W_{0}+\mathrm{RPA}$, and $G_{0} W_{0}+\mathrm{BSE}$ approach are shown in Figs. 6 and 7. For comparison, the imaginary part of the dielectric function acquired at the PBE+RPA and $G_{0} W_{0}+\mathrm{RPA}$ levels represents that without inclusion of the $e-e$ interaction, and that with inclusion of the $e-e$ interaction led to a redshift; however, the overall shape of the spectra was conserved. With the involvement of the $e-h$ interactions (i.e., excitonic effects) in the BSE, a significant blueshift of $\operatorname{Im}(\omega)$ is produced. In addition, the most important physical effect with the inclusion of the $e-h$ interactions was the appearance of some bound excitons below the $G W$ gap [38]. It is known that the real part of the dielectric function shows the material's polarizability. The static values of the real part of the complex dielectric function at $\omega=0$ depicted in Fig. 6(a) are 2.40 (with $G W+\mathrm{BSE}$ ), 3.12 (with $G W+\mathrm{RPA}$ ), and 3.25 (with PBE). The inclusion of $e$-e interactions with $G W+\mathrm{RPA}$ and $e$ - $h$ interactions with $G W+$ BSE decreases the static dielectric constant $(\omega=0)$ compared to PBE+RPA; see Fig. 6. From the real part of the dielectric function, negative values appear at $\approx 4 \mathrm{eV}$.
This shows the metallic character of the HG monolayer in the ultraviolet (UV) part of the electromagnetic spectrum. Also, we have compared the imaginary part of the dielectric function with two PBE+RPA methods, namely $G W+$ RPA and $G W+\mathrm{BSE}$, in which $G W$ plus BSE peaks shifted toward a higher photon energy range compared to PBE as shown in Fig. 6(b).

Figure 6(c) illustrates the computed refractive index of a HG monolayer using PBE, and $G W$ plus BSE. The value of the static refraction index $n(0)$ in the HG sheet is 1.54 and 1.81 using $G W$ plus BSE and PBE, respectively. It is well known that the $G W$ plus BSE method gives very accurate optical properties. According to that method, the refractive index of a HG monolayer is 1.54 . This means that the HG monolayer is a nearly transparent material because its refractive index is equal to the glass refractive index. From Fig. 6(c), we can observe that at energies about $4 \mathrm{eV}$ using both methods, the refractive index is minimum and at that value the absorption is maximum. The refractive index $n$ increases with photon energy in the infrared (IR) region, while it decreases monotonically in the visible and UV region and then it steadily decreases. The maximum refractive index is found to be 1.95 at $1.28 \mathrm{eV}$ for a $\mathrm{HG}$ monolayer with the $G W$ plus BSE method. These refractive indices could enable HG monolayer to be used as an optical cavity layer [68]. Additionally, we have calculated the extinction coefficient $K$ 
as shown in Fig. 6(d). The values of the extinction coefficient $K$ of a HG monolayer rapidly decrease with increasing photon energy in the UV region and become an oscillatory type of behavior after $5 \mathrm{eV}$. The maximum values of the extinction coefficient are found at $4 \mathrm{eV}$. This means that at these energies the photons will be absorbed very fast (i.e., penetration depth will be the shortest at a particular photon energy).

Now, we discuss further the optical absorption spectra shown in Fig. 7(a). The absorption spectra of a HG monolayer are computed by the PBE and $G W$ plus BSE methods. When the PBE+RPA were included, the entire absorption shifted to the lower photon energy, i.e., it showed a redshift, while with $e-h$ interactions included (i.e., $G W+\mathrm{BSE}$ ), the whole spectrum is shifted toward higher photon energy. Henceforth, the blueshift of optical absorption occurs due to the excitonic effects. We can see that the absorption starts from a low photon energy of $0.65 \mathrm{eV}$ with PBE plus RPA and a slightly shifted higher energy at $1.28 \mathrm{eV}$ with $G W+\mathrm{BSE}$ methods. The absorption coefficient of a HG monolayer reaches its maximum value of 3.5 and $4 \mathrm{eV}$ with PBE+RPA and $G W+\mathrm{BSE}$, respectively. We found the maximum values of optical conductivity at the same photon energy (like the maximum optical absorption), as shown in Fig. 7(b). After $4 \mathrm{eV}$ of photon energy, the optical absorption and conductivity are drastically decreased with increasing photon energy, and they show oscillatory behavior. Also, the electron energy loss spectra (EELS) $(L)$ are very useful in realizing plasma resonance phenomena as distinct from normal interband transitions. Figure $7(\mathrm{c})$ shows the $L$ of a HG monolayer with two methods. Furthermore, there is a large sharp peak in photon energy at $1.52 \mathrm{eV}$ with the $G W+\mathrm{BSE}$ method, and the same peak was found at very low photon energy with the PBE+RPA method. This peak at lower photon energy comes from the Frenkel exciton $[69,70]$, which is related to $\pi$ plasmon in $L$. The second strong and sharp peak appears at $5.5 \mathrm{eV}$, which is strongly related to $\pi+\sigma$ electron plasmon [60]. The reflectivity of a HG monolayer is presented in Fig. 7(d). It can be seen that the reflectivity in a HG monolayer shows more of an oscillatory nature up to $5.5 \mathrm{eV}$, and after that it rapidly decreases with increasing photon energy. The maximum reflectivity is found to be $18 \%$ around $4 \mathrm{eV}$. The reflectivity values do not exceed $20 \%$ in the whole region, which is why it can be used in antireflective coating. In contrast, the reflectivity was found to be $40 \%$ in monolayer graphene by Qiu and co-workers [71], which is two times larger than the HG monolayer.

\section{Thermoelectric properties}

In this section, we will discuss the thermoelectric properties such as the Seebeck coefficient $\alpha$, electrical conductivity $\sigma$, electronic thermal conductivity $\kappa_{e}$, thermopower, PF, and electronic figure of merit, $Z T$, as a function of chemical potential for a HG monolayer. During the calculation of thermal and electrical conductivity, a constant relaxation time approximation with $\tau=10^{-14} \mathrm{~s}$ has been used. The thermopower $\alpha$ of the HG monolayer as a function of chemical potential $\mu$ is presented in Fig. 8(a) at two different temperatures, 300 and $800 \mathrm{~K}$. The values of $\mu$ in the negative and positive side show $p$-type and $n$-type doping in the system, respectively. Each term of the thermoelectric properties

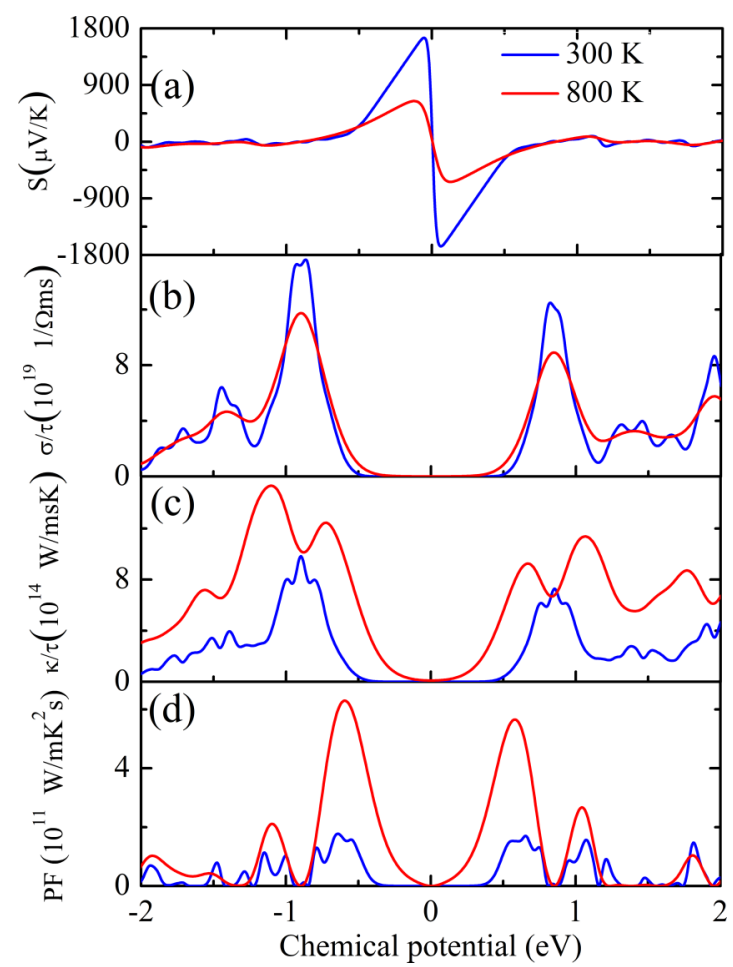

FIG. 8. Thermoelectric components as a function of chemical potential in the range of -2 to $+2 \mathrm{eV}$. (a) Seebeck coefficient, (b) electrical conductivity, (c) electronic thermal conductivity, and (d) power factor of a HG monolayer. The electronic thermal and electrical conductivity is calculated with a constant relaxation time $\left(\tau=10^{-14} \mathrm{~s}\right)$.

had enhanced characteristics in $p$-type and $n$-type doping in the HG monolayer. From Fig. 8(a), the sharp values of thermopower are observed around the Fermi level (i.e., the $p$ type and $n$-type doping side) at $300 \mathrm{~K}$, and they got suppressed at higher temperature, which indicates that an optimal carrier concentration is favorable for enhancing thermoelectric performance. The maximum values of thermopower at room temperature are found to be $1662.59 \mu \mathrm{V} / \mathrm{K}$ in $n$-type doping and $645.67 \mu \mathrm{V} / \mathrm{K}$ in $p$-type doping at $800 \mathrm{~K}$. These values are higher than most of the flat materials of the graphene family. Graphene, $\alpha, \beta$, and $(6,6,12)$-graphyne show nearly the same Seebeck coefficient as nearly $135 \mu \mathrm{V} / \mathrm{K}$, and $\gamma$ graphyne shows a relatively higher value around $600 \mu \mathrm{V} / \mathrm{K}$ [72,73]. This remarkable enhancement can be ascribed to the quantum confinement effect, which induces sharp DOS peaks near the Fermi energy, as can be seen in Fig. 3. The thermopower graph has almost similar values on both $p$-type and $n$-type doping due to the symmetric nature of valence and conduction bands (see Fig. 2). The electrical conductivity is depicted in Fig. 8(b), which shows the values of $\sigma / \tau$ as a function of chemical potential with increasing behavior near the Fermi level. The increment in the thermopower correlates with the gradual decrease in electrical conductivity in $p$-type and $n$-type doping, which clearly reflects the electronic band structure in Fig. 2(a). The electrical conductivity is higher in the positive chemical potential region than in the negative region. This suggests that the electron doping in HG will be 
more advantageous for thermoelectric performance than hole doping.

Furthermore, the electrical conductivity is also sensitive to temperature similar to the thermopower, $\sigma / \tau$, which exhibits a relatively lower value at $800 \mathrm{~K}$ with an enhanced value near the Fermi level [see Fig. 8(b)]. It is also noted that raising the temperature decreases the electrical conductivity, which can be explained by the increasing carrier concentration plus collisions and scattering phenomena at high temperatures. In addition, the variation of electronic thermal conductivity with chemical potential is presented in Fig. 8(c). The electronic thermal conductivity shows higher sensitivity with temperature and follows the inverse trend to that of electrical conductivity as in Fig. 8(b). On the other hand, it shows that the values of $\kappa_{e}$ are relatively higher in $p$-type doping in a HG monolayer similar to electric conductivity.

The interplay between sufficiently large electrical conductivity (higher $\mu$ ) and a sharp peak in the Seebeck coefficient near the Fermi energy (smaller $\mu$ ) results in a high power factor, which is critical to good thermoelectric performance. The power factor, PF $\left(\sigma S^{2}\right)$, of a $\mathrm{HG}$ monolayer is shown in Fig. 8(d). The computed values of $\sigma \alpha^{2}$ at two different temperatures are depicted as a function of chemical potential. The maximum PF mainly increases with a band-gap decrease, which could be associated with the increased electrical conductivity [74]. Similar to previous results, the PF also shows greater values in $p$-type doping as compared to $n$-type doping at higher temperature, and it increases with an increase in the doping level. Additionally, the sharp electronic density of states near the Fermi level or flat band lines in the electronic band structure near the Fermi level enhanced the thermopower $\alpha$. Furthermore, the high DOS near the Fermi energy provides large electrical conductivity.

The directional dependence of thermopower $S$, electrical conductivity $\sigma$, electronic thermal conductivity $\kappa_{e}$, and $\mathrm{PF}$ as a function of chemical potential are shown in Fig. 9. The thermopower depends on the sharp DOS near the Fermi level and is independent of the dispersion of the band line. That is why the thermopower values in the $a$ - and $b$-direction are the same [see Fig. 9(a)]. This calculated feature is comparable with typical thermoelectric materials [75], while the electrical and electronic thermal conductivities [see Figs. 9(b) and 9(c)] show a significant direction dependence, which is in good agreement with the $G-X$ and $G-Y$ band lines in Fig. 2 . Direction dependence in the power factor comes from the proportionality of the electrical conductivity. In addition to this, the descriptions of these thermoelectric coefficients (such as the Seebeck coefficient, electrical conductivity, electronic thermal conductivity, and power factor) as a function of carrier concentrations can be obtained in Ref. [76].

For high-performance thermoelectric applications, the values of the figure of merit $(Z T)$ should be high. The $Z T$ value depends on $S, \sigma$, and $\kappa$, in which $Z T$ is inversely proportional to $\kappa\left(=\kappa_{e}+\kappa_{l}\right)$. In fact, the small values of lattice thermal conductivity significantly enhanced the $Z T$ value. Recently, it was reported that lattice thermal conductivity is very small ( 6.0 and $14.1 \mathrm{~W} / \mathrm{mK}$ in the $a$-direction and the $b$-direction, respectively) in holey graphene [49] as compared to graphene (2000-5000 W/mK) [77,78]. According to that, the electronic $Z T$ values change by small values. From all the transport

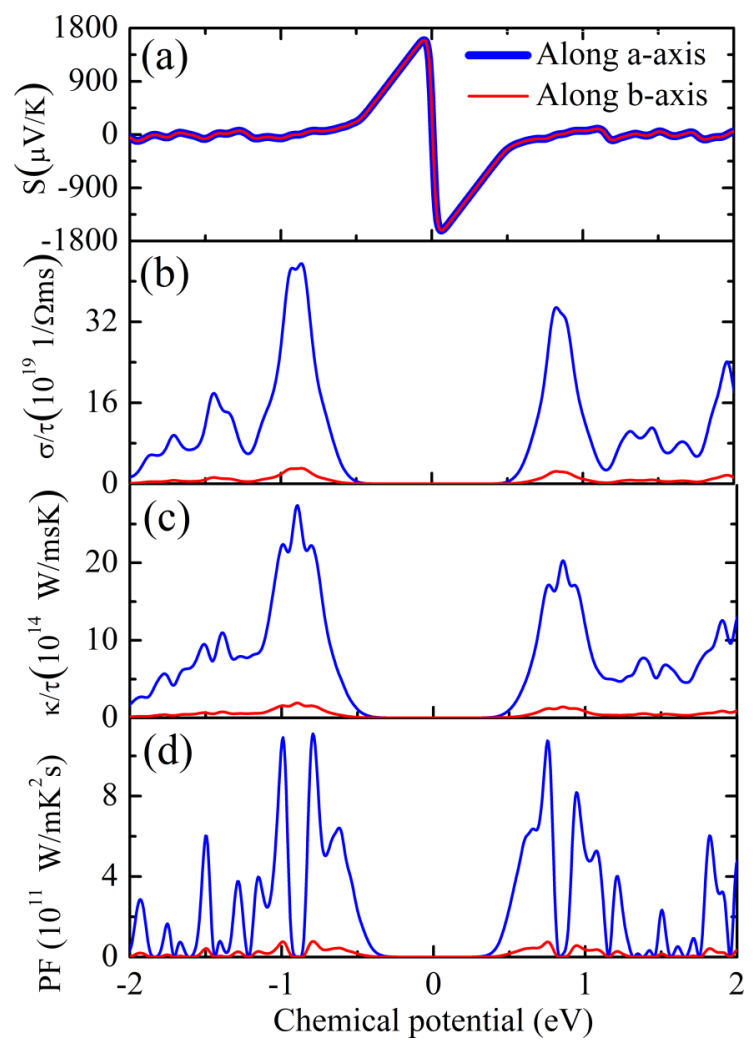

FIG. 9. The directional dependence of the thermoelectric component, i.e., $a$-direction and $b$-direction, as a function of chemical potential in the range of -2 to $+2 \mathrm{eV}$. (a) Seebeck coefficient, (b) electrical conductivity, (c) electronic thermal conductivity, and (d) power factor of a HG monolayer. The electronic thermal and electrical conductivity is calculated with a constant relaxation time $\left(\tau=10^{-14} \mathrm{~s}\right)$.

quantities, the calculated electronic figure of merit, $Z T$, as a function of chemical potential, at fixed temperatures of 300 and $800 \mathrm{~K}$ for a HG monolayer, is shown in Fig. 10(a). At room temperature, $Z T$ reaches a maximum in $p$-type doping levels, and a corresponding value of 1.13 is observed at the chemical potential $-0.40 \mathrm{eV}$. At higher temperature $(800 \mathrm{~K})$, the $Z T$ value goes down to 0.89 at a chemical potential $\pm 0.22 \mathrm{eV}$ for both $p$ - and $n$-type doping. Additionally, the variation of $Z T_{e}$ with respect to carrier concentrations can be obtained in Ref. [76]. Our calculated $Z T$ value is higher than most of the $2 \mathrm{D}$ material. Previously, the values of $Z T$ were reported as 1.02 for boron monochalcogenide [53], $\approx 0.38$ in $\mathrm{CP}$ monolayer [79], $\approx 0.75$ for arsenene monolayer [80], $\approx 0.78$ for antimonene monolayer [80], 0.08 for a single layer of graphene [81], and 0.12 for $\beta-, 0.03$ for $\alpha-, 0.05$ for $(6,6,12)$-, and 0.17 for $\gamma$-graphyne $[72,73]$. It was also seen that the figure of merit $Z T$ value is more than 5 in nitrogenated holey graphene [82]. Furthermore, it is evident from Fig. 10(b) that the electronic figure of merit exhibits isotropic behavior.

\section{CONCLUSION}

In summary, we systematically investigated the electronic, optical, and thermoelectric properties of a recently synthesized novel 2D planar HG monolayer by using first-principles 


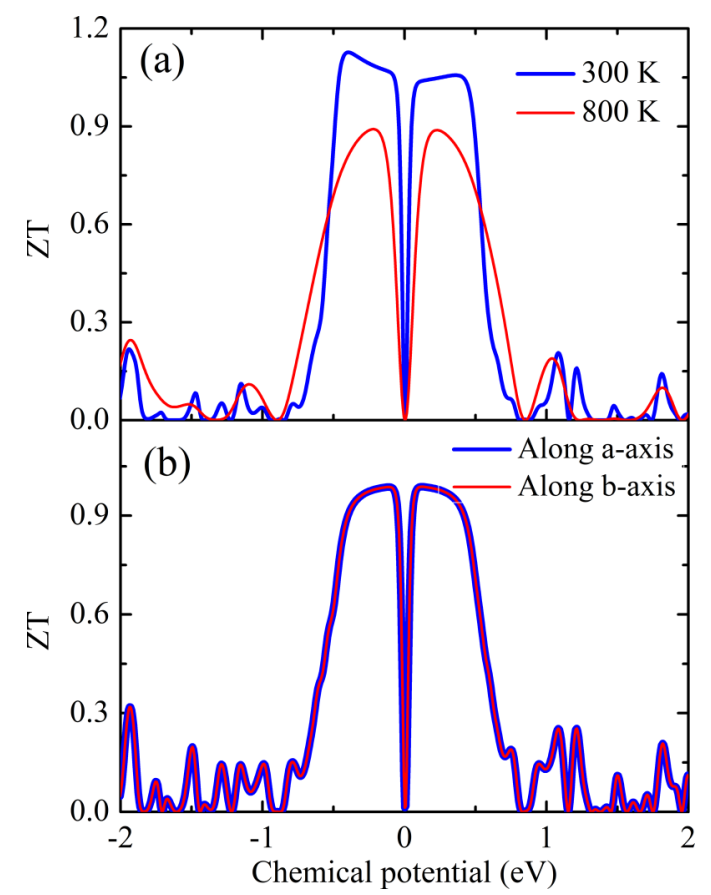

FIG. 10. Electronic figure of merit, ZT: (a) average and (b) $a$ direction and $b$-direction of a $\mathrm{HG}$ monolayer. The directionaldependent electronic figure of merit along the $a$ - and $b$-directions is plotted at $300 \mathrm{~K}$. calculations. HG exhibits a direct band gap with anisotropic electron and hole mobilities. HSE06 predicts an accurate $0.95 \mathrm{eV}$ band gap close to the experimental value. To find the accurate optical properties, we have used $G W$ plus BSE methods. The evaluated optical gap is $1.28 \mathrm{eV}$. Also, the excitonic effects play a crucial role in the optical properties, with a significantly small $0.08 \mathrm{eV}$ binding energy. A broad absorption spectrum is found in the visible light region. Interestingly, the reflectivity does not exceed more than $20 \%$, which is lower than that of pristine graphene. Therefore, it is interpreted that this material can be used as an antireflective coating material. Moreover, we found superior thermopower values of $1662.59 \mu \mathrm{V} / \mathrm{K}$ and a corresponding electronic figure of merit of 1.13 in HG monolayer, which is greater than most of the 2D materials due to the quantum confinement effect of the nanopores. We suggest that monolayer HG possesses extraordinary electronic, optical, and thermoelectric properties. Our results are timely, and they suggest that HG may be suitable for optoelectronic and thermoelectric device applications.

\section{ACKNOWLEDGMENTS}

D.S. and R.A. thank Olle Engkvists Stiftelse, Carl Tryggers Stiftelse for Vetenskaplig Forskning (CTS), and the Swedish Research Council (VR-2016-06014) for financial support. SNIC and HPC2N are acknowledged for providing the computing facilities.
[1] K. S. Novoselov, A. K. Geim, S. V. Morozov, D. Jiang, Y. Zhang, S. V. Dubonos, I. V. Grigorieva, and A. A. Firsov, Science 306, 666 (2004).

[2] A. K. Geim and K. S. Novoselov, Nanoscience and Technology: A Collection of Reviews from Nature Journals (World Scientific, Singapore, 2010), pp. 11-19.

[3] C. Berger, Z. Song, T. Li, X. Li, A. Y. Ogbazghi, R. Feng, Z. Dai, A. N. Marchenkov, E. H. Conrad, P. N. First, and W. A. de Heer, J. Phys. Chem. B 108, 19912 (2004).

[4] M. Liu, X. Yin, E. Ulin-Avila, B. Geng, T. Zentgraf, L. Ju, F. Wang, and X. Zhang, Nature (London) 474, 64 (2011).

[5] B. Liu and K. Zhou, Prog. Mater. Sci. 100, 99 (2019).

[6] H. C. Lee, W.-W. Liu, S.-P. Chai, A. R. Mohamed, A. Aziz, C.-S. Khe, N. S. Hidayah, and U. Hashim, RSC Adv. 7, 15644 (2017).

[7] A. A. Balandin, S. Ghosh, W. Bao, I. Calizo, D. Teweldebrhan, F. Miao, and C. N. Lau, Nano Lett. 8, 902 (2008).

[8] A. C. Neto, F. Guinea, N. M. Peres, K. S. Novoselov, and A. K. Geim, Rev. Mod. Phys. 81, 109 (2009)

[9] G. Liu, W. Jin, and N. Xu, Chem. Soc. Rev. 44, 5016 (2015).

[10] K. S. Novoselov and A. Geim, Nat. Mater 6, 183 (2007).

[11] K. S. Novoselov, A. K. Geim, S. V. Morozov, D. Jiang, M. I. Katsnelson, I. Grigorieva, S. Dubonos, and A. Firsov, Nature (London) 438, 197 (2005).

[12] I. Meric, M. Y. Han, A. F. Young, B. Ozyilmaz, P. Kim, and K. L. Shepard, Nat. Nanotech. 3, 654 (2008).
[13] C. Lee, X. Wei, J. W. Kysar, and J. Hone, Science 321, 385 (2008).

[14] L. Zhang, Y. Zhang, J. Camacho, M. Khodas, and I. Zaliznyak, Nat. Phys. 7, 953 (2011).

[15] F. Schwierz, Nat. Nanotech. 5, 487 (2010).

[16] Z. Yang and J. Hao, J. Mater. Chem. C 4, 8859 (2016).

[17] A. Gupta, T. Sakthivel, and S. Seal, Prog. Mater. Sci. 73, 44 (2015).

[18] K. Momeni, Y. Ji, Y. Wang, S. Paul, S. Neshani, D. E. Yilmaz, Y. K. Shin, D. Zhang, J.-W. Jiang, H. S. Park et al., npj Comput. Mater. 6, 22 (2020).

[19] D. W. Boukhvalov and M. I. Katsnelson, J. Phys.: Condens. Matter 21, 344205 (2009).

[20] P. P. Shinde and V. Kumar, Phys. Rev. B 84, 125401 (2011).

[21] K. J. Jeon, Z. Lee, E. Pollak, L. Moreschini, A. Bostwick, C.-M. Park, R. Mendelsberg, V. Radmilovic, R. Kostecki, T. J. Richardson, and E. Rotenberg, ACS Nano 5, 1042 (2011).

[22] K. E. Whitener, J. Vac. Sci. Technol. A 36, 05G401 (2018).

[23] A. Guirguis, J. W. Maina, L. Kong, L. C. Henderson, A. Rana, L. H. Li, M. Majumder, and L. F. Dumée, Carbon 155, 660 (2019).

[24] C. Backes, A. M. Abdelkader, C. Alonso, A. Andrieux-Ledier, R. Arenal, J. Azpeitia, N. Balakrishnan, L. Banszerus, J. Barjon, R. Bartali et al., 2D Mater. 7, 022001 (2020).

[25] P. Liu, A. T. Liu, D. Kozawa, J. Dong, J. F. Yang, V. B. Koman, M. Saccone, S. Wang, Y. Son, M. H. Wong et al., Nat. Mater. 17, 1005 (2018). 
[26] A. Guirguis, J. W. Maina, X. Zhang, L. C. Henderson, L. Kong, H. Shon, and L. F. Dumée, Mater. Horiz. 7, 1218 (2020).

[27] A. Cupo, P. Masih Das, C. C. Chien, G. Danda, N. Kharche, D. Tristant, M. Drndić, and V. Meunier, ACS Nano 11, 7494 (2017).

[28] L. Shao, G. Chen, H. Ye, Y. Wu, H. Niu, and Y. Zhu, J. Appl. Phys. 116, 113704 (2014).

[29] P. Masih Das, J. P. Thiruraman, Y.-C. Chou, G. Danda, and M. Drndić, Nano Lett. 19, 392 (2018).

[30] M. S. H. Boutilier, D. Jang, J.-C. Idrobo, P. R. Kidambi, N. G. Hadjiconstantinou, and R. Karnik, ACS Nano 11, 5726 (2017).

[31] C. Sun, B. Wen, and B. Bai, Sci. Bull. 60, 1807 (2015).

[32] Y. Yang, X. Yang, L. Liang, Y. Gao, H. Cheng, X. Li, M. Zou, R. Ma, Q. Yuan, and X. Duan, Science 364, 1057 (2019).

[33] K. Xu, J. I. Urgel, K. Eimre, M. Di Giovannantonio, A. Keerthi, H. Komber, S. Wang, A. Narita, R. Berger, P. Ruffieux, C. A. Pignedoli, J. Liu, K. Müllen, R. Fasel, and X. Feng, J. Am. Chem. Soc. 141, 7726 (2019).

[34] T. G. Pedersen, C. Flindt, J. Pedersen, N. A. Mortensen, A.-P. Jauho, and K. Pedersen, Phys. Rev. Lett. 100, 136804 (2008).

[35] J. Eroms and D. Weiss, New J. Phys. 11, 095021 (2009).

[36] A. Sinitskii and J. M. Tour, J. Am. Chem. Soc. 132, 14730 (2010).

[37] W. Oswald and Z. Wu, Phys. Rev. B 85, 115431 (2012).

[38] C. Moreno, M. Vilas-Varela, B. Kretz, A. Garcia-Lekue, M. V. Costache, M. Paradinas, M. Panighel, G. Ceballos, S. O. Valenzuela, D. Peña et al., Science 360, 199 (2018).

[39] P. Hohenberg and W. Kohn, Phys. Rev. 136, B864 (1964).

[40] G. Kresse and J. Furthmüller, Phys. Rev. B 54, 11169 (1996).

[41] J. Heyd, G. E. Scuseria, and M. Ernzerhof, J. Chem. Phys. 118, 8207 (2003).

[42] G. Henkelman, A. Arnaldsson, and H. Jónsson, Comput. Mater. Sci. 36, 354 (2006).

[43] M. Shishkin and G. Kresse, Phys. Rev. B 74, 035101 (2006).

[44] F. Karlicky and M. Otyepka, J. Chem. Theor. Comput. 9, 4155 (2013).

[45] D. Y. Qiu, F. H. da Jornada, and S. G. Louie, Phys. Rev. Lett. 111, 216805 (2013).

[46] M. Shishkin and G. Kresse, Phys. Rev. B 75, 235102 (2007).

[47] G. K. Madsen and D. J. Singh, Comput. Phys. Commun. 175, 67 (2006).

[48] T. Hussain, E. Olsson, K. A. Alhameedi, Q. Cai, and A. Karton, J. Phys. Chem. C 124, 9734 (2020).

[49] B. Mortazavi, M. E. Madjet, M. Shahrokhi, S. Ahzi, X. Zhuang, and T. Rabczuk, Carbon 147, 377 (2019).

[50] G. Calogero, N. R. Papior, B. Kretz, A. Garcia-Lekue, T. Frederiksen, and M. Brandbyge, Nano Lett. 19, 576 (2018).

[51] E. Tiras, S. Ardali, T. Tiras, E. Arslan, S. Cakmakyapan, O. Kazar, J. Hassan, E. Janzèn, and E. Ozbay, J. Appl. Phys. 113, 043708 (2013).

[52] J. Bardeen and W. Shockley, Phys. Rev. 80, 72 (1950).

[53] P. Mishra, D. Singh, Y. Sonvane, and R. Ahuja, Sustain. Energy Fuels 4, 2363 (2020).

[54] J. Xi, M. Long, L. Tang, D. Wang, and Z. Shuai, Nanoscale 4, 4348 (2012).
[55] G. Wang, R. Pandey, and S. P. Karna, Nanoscale 8, 8819 (2016).

[56] R. Fei and L. Yang, Nano Lett. 14, 2884 (2014).

[57] J. Qiao, X. Kong, Z.-X. Hu, F. Yang, and W. Ji, Nat. Commun. 5, 4475 (2014).

[58] J. Chen, J. Xi, D. Wang, and Z. Shuai, J. Phys. Chem. Lett. 4, 1443 (2013).

[59] D. Li, Z.-G. Shao, Q. Hao, and H. Zhao, J. Appl. Phys. 115, 233701 (2014).

[60] M. Shahrokhi, Appl. Surf. Sci. 390, 377 (2016).

[61] L. Yang, Phys. Rev. B 83, 085405 (2011).

[62] S. Huang, Y. Liang, and L. Yang, Phys. Rev. B 88, 075441 (2013).

[63] V. Tran, R. Fei, and L. Yang, 2D Mater. 2, 044014 (2015).

[64] B. Ul Haq, S. Al Faify, R. Ahmed, F. K. Butt, A. Laref, and M. Shkir, Phys. Rev. B 97, 075438 (2018).

[65] D. Singh, S. K. Gupta, Y. Sonvane, and I. Lukačević, J. Mater. Chem. C 4, 6386 (2016).

[66] M. Gajdoš, K. Hummer, G. Kresse, J. Furthmüller, and F. Bechstedt, Phys. Rev. B 73, 045112 (2006).

[67] E. E. Salpeter and H. A. Bethe, Phys. Rev. 84, 1232 (1951).

[68] A. B. Matsko, Practical Applications of Microresonators in Optics and Photonics (CRC, Boca Raton, FL, 2009).

[69] D. S. Ellis, J. P. Hill, S. Wakimoto, R. J. Birgeneau, D. Casa, T. Gog, and Y.-J. Kim, Phys. Rev. B 77, 060501(R) (2008).

[70] E. Baldini, L. Chiodo, A. Dominguez, M. Palummo, S. Moser, M. Yazdi-Rizi, G. Auböck, B. P. Mallett, H. Berger, A. Magrez et al., Nat. Commun. 8, 13 (2017).

[71] B. Qiu, X. Zhao, G. Hu, W. Yue, J. Ren, and X. Yuan, Nanomaterials 8, 962 (2018).

[72] H. Sevinçli and C. Sevik, Appl. Phys. Lett. 105, 223108 (2014).

[73] M. Ali, X. Pi, Y. Liu, and D. Yang, Nanotechnology 29, 045402 (2017).

[74] J. Y. Bi, L. H. Han, Q. Wang, L. Y. Wu, R. Quhe, and P.-F. Lu, Chin. Phys. B 27, 026802 (2018).

[75] L. D. Zhao, S. H. Lo, Y. Zhang, H. Sun, G. Tan, C. Uher, C. Wolverton, V. P. Dravid, and M. G. Kanatzidis, Nature (London) 508, 373 (2014).

[76] See Supplemental Material at http://link.aps.org/supplemental/ 10.1103/PhysRevB.102.075444 for the Seebeck coefficient, electrical conductivity, electronic thermal conductivity, power factor, and electronic figure of merit as a function of carrier concentrations.

[77] B. D. Kong, S. Paul, M. B. Nardelli, and K. W. Kim, Phys. Rev. B 80, 033406 (2009).

[78] Y. Anno, Y. Imakita, K. Takei, S. Akita, and T. Arie, 2D Mater. 4, 025019 (2017).

[79] D. Singh, S. Kansara, S. K. Gupta, and Y. Sonvane, J. Mater. Sci. 53, 8314 (2018).

[80] S. Sharma, S. Kumar, and U. Schwingenschlögl, Phys. Rev. Applied 8, 044013 (2017).

[81] A. Reshak, S. A. Khan, and S. Auluck, J. Mater. Chem. C 2, 2346 (2014).

[82] Y. Zhao, Z. Dai, C. Lian, and S. Meng, RSC Adv. 7, 25803 (2017). 